\title{
Cultura de segurança do paciente em unidade de terapia intensiva: perspectiva da equipe de enfermagem*
}

Janeide Freitas Mello ${ }^{1}$, Sayonara Fátima Faria Barbosa ${ }^{2}$

\footnotetext{
* Artigo extraído da Dissertação de Mestrado "Cultura de segurança do paciente em unidade de terapia intensiva, perspectiva da enfermagem, apresentada ao Programa de Pós-Graduação em Enfermagem da Universidade Federal de Santa Catarina, SC, Brasil.

${ }^{1}$ Enfermeira, Mestre em Enfermagem. Discente do Programa de Pós-Graduação em Enfermagem, nível Doutorado, da Universidade Federal de Santa Catarina. Enfermeira do Hospital Universitário da Universidade Federal de Santa Catarina. Florianópolis, SC, Brasil. E-mail: janeidef@gmail.com.

${ }^{2}$ Enfermeira, Doutora em Ciências da Saúde. Professora Adjunto da Universidade Federal de Santa Catarina. Florianópolis, SC, Brasil. E-mail: sayonara.barbosa@ufsc.br.
}

Recebido: 18/08/2016.

Aceito: 08/12/2016.

Publicado: 04/05/2017.

Como citar esse artigo:

Mello JF, Barbosa SFF. Cultura de segurança do paciente em unidade de terapia intensiva: perspectiva da equipe de enfermagem. Rev. Eletr. Enf. [Internet]. 2017 [acesso em: _____ _ _ 19:a07. Disponível em: http://dx.doi.org/10.5216/ree.v19.38760.

\section{RESUMO}

Survey realizado com objetivo de identificar e comparar as dimensões da cultura de segurança do paciente na perspectiva dos profissionais de enfermagem de duas Unidades de Terapia Intensiva. Foi aplicado o Hospital Survey on Patient Safety Culture (HSOPSC) entre abril a junho de 2011, o qual foi respondido por 86 profissionais, equivalente a $(83,49 \%)$ da população de duas Unidades de Terapia Intensiva. As dimensões com maior avaliação positiva foram trabalho em equipe nas unidades (62,8\%), expectativas e ações para promoção da segurança do supervisor (49\%) e aprendizado organizacional $(46,1 \%)$. As dimensões com menor avaliação foram apoio da gestão para a segurança $(13,6 \%)$, respostas não punitivas aos erros $(17,5 \%)$ e percepção geral de segurança $(25,9 \%)$. Os resultados indicam que a cultura de segurança precisa ser desenvolvida nos locais do estudo, com especial atenção aquelas com avaliação menos positiva sobre a cultura de segurança.

Descritores: Segurança do Paciente; Cultura; Unidades de Terapia Intensiva; Enfermagem.

\section{INTRODUÇÃO}

A segurança do paciente tem sido uma das principais preocupações das organizações de saúde para melhorar a qualidade de seus serviços. Em resposta as recomendações para a melhoria da segurança do paciente do Institute of Medicine (IOM) em $2000^{(1)}$, a segurança do paciente tornou-se uma política de saúde prevalente em todo o mundo; sendo vista como um paradigma, prática ou movimento reunindo diferentes comunidades a um objetivo comum ${ }^{(2)}$. 
Assim, sob o imperativo da segurança do paciente diversas estratégias têm sido implementadas para reduzir erros na assistência em saúde ${ }^{(3-4)}$. No entanto, há o reconhecimento de que a principal barreira para uma assistência segura não é a falta de dados, conhecimentos ou experiência dos profissionais, mas o fato que muitas organizações de saúde têm culturas fragilizadas que reforçam comportamentos negativos. Estas culturas dificultam a promoção de cuidado de alta qualidade, atendimento eficaz, de forma eficiente e rentável. Sendo assim, a cultura da organização de saúde é incapaz de se adaptar para atender às necessidades e limitações e de proporcionar um ambiente de trabalho estimulante e seguro ${ }^{(5)}$.

O desenvolvimento de uma cultura de segurança do paciente foi uma das recomendações feitas pelo IOM para ajudar as instituições de saúde a melhorar a segurança ${ }^{(1)}$. A cultura de segurança de uma organização são os valores compartilhados entre os seus membros sobre o que é importante, suas crenças sobre como as coisas funcionam na organização, e a interação destes com a unidade de trabalho, as estruturas e sistemas organizacionais, que juntos, produzem normas de comportamento que promovem a segurança( ${ }^{(6)}$.

Há tendências emergentes que correlacionam a cultura de segurança do paciente com resultados favoráveis para os pacientes tais como diminuição das taxas de readmissão e mortalidade, satisfação do paciente e lesão por pressão. Quando se trata de cultura de segurança do paciente em Unidade de Terapia Intensiva (UTI) a mortalidade e a satisfação familiar apresentam correlações ainda mais significativas ${ }^{(7)}$. Além disso, uma cultura de segurança positiva, também pode estar relacionada a uma menor taxa de eventos $\operatorname{adversos}^{(8)}$.

Nesse sentido, a cultura de segurança nos ambientes de cuidado de saúde contribui para a qualidade e segurança do paciente. Os elementos principais de uma cultura de segurança incluem liderança eficaz, trabalho em equipe, aprendizagem a partir dos erros e criação de um ambiente de confiança ${ }^{(9)}$. No entanto, a maioria das organizações de saúde possuem uma estrutura hierárquica ultrapassada, que inibe a comunicação, compromete a participação plena e prejudica o trabalho em equipe ${ }^{(5)}$.

Diante disso, a avaliação da cultura de segurança do paciente é o primeiro passo para melhorar a segurança do paciente nas organizações de saúde por permitir: uma visão clara dos aspectos de segurança dos pacientes que requerem atenção urgente; identificação de pontos fortes e fracos de sua cultura de segurança; detecção da existência de problemas de segurança do paciente nas unidades e avaliação comparativa dos seus escores como outras instituições de saúde ${ }^{(10)}$.

Para avaliar a cultura de segurança, vários instrumentos foram desenvolvidos ${ }^{(11)}$, geralmente na forma de questionários quantitativos baseados na combinação de diversas dimensões da cultura de segurança do paciente. As dimensões mais frequentemente citadas incluem: compromisso da liderança para a segurança, comunicação aberta baseada na confiança, aprendizagem organizacional, abordagem não punitiva ao relato de eventos adversos, trabalho em equipe e crença compartilhada na importância da segurança ${ }^{(12)}$.

Portanto, considerando a importância de avaliar a cultura de segurança do paciente, este estudo consistiu na aplicação do Hospital Survey on Patient Safety Culture (HSOPSC), um instrumento de avaliação 
da cultura de segurança criado pela Agency for Healthcare Research and Quality (AHRQ) dos Estados Unidos da América (EUA) $)^{(13)}$. Por meio da aplicação desse instrumento, este estudo teve o objetivo de identificar e comparar as dimensões da cultura de segurança do paciente sob a ótica dos profissionais de enfermagem de duas UTI adulto.

\section{MÉTODO}

Estudo quantitativo, tipo survey transversal, realizado em duas UTI adulto de hospitais públicos do sul do Brasil, no período de abril a junho de 2011. Para a realização do estudo foram adotadas as normas brasileiras para pesquisa com seres humanos sendo o projeto de pesquisa aprovado pelos Comitês de Ética em Pesquisa com Seres Humanos de ambas as instituições mediante protocolos no 59/10 de 25/10/2010 e ำ 1.113 de 29/11/2010.

Na composição dos participantes, adotou-se a amostragem de conveniência. A população $(N)$ do estudo foi constituída por 109 profissionais de enfermagem que tiveram como critério de inclusão um tempo de atuação de no mínimo seis meses nas UTI onde o estudo foi realizado. Dos 109 profissionais, cinco foram excluídos do estudo por se encontrarem afastados do trabalho durante o período de coleta de dados e um se recusou a participar do estudo. Assim, 103 participantes (n) receberam os formulários, e 97 realizaram a devolução.

A coleta de dados consistiu na aplicação do HSOPSC, instrumento cuja adaptação transcultural e validação para a língua portuguesa no Brasil foram concluídos em $2013^{(14)}$. Como o instrumento utilizado ainda estava em processo de validação, quando da realização do presente estudo, optou-se pela utilização da versão traduzida para a língua portuguesa do $\operatorname{HSOPSC}^{(15)}$, que foi submetida à pré-teste com cinco profissionais. A versão do estudo, comparada com a versão submetida a adaptação e validação ${ }^{(14)}$, não apresentou diferenças em sua redação que comprometesse a compreensão do instrumento, e por conseguinte a avaliação realizada pelo profissional.

O HSOPSC está disponível para domínio público desde 2004 e vem sendo amplamente utilizado devido as suas propriedades psicométricas favoráveis ${ }^{(16)}$, evidenciadas pelos seus atributos de confiabilidade e validade.

O instrumento contém 42 questões agrupadas em 12 dimensões: trabalho em equipe dentro das unidades; expectativas e ações de promoção da segurança do paciente do supervisor/gerente; aprendizado organizacional, melhoria contínua; feedback e comunicação a respeito de erros; abertura para comunicações; pessoal; respostas não punitivas aos erros; apoio da gestão hospitalar para a segurança do paciente; trabalho em equipe entre as unidades do hospital; transferências internas e passagens de plantão; percepção geral de segurança do paciente e frequência de eventos relatados.

Cada uma das 12 dimensões do HSOPSC inclui três ou quatro itens avaliados por uma escala Likert de cinco pontos com categorias de respostas em termos de concordância (nove dimensões) ou de frequência (três dimensões). A avaliação de cada dimensão é atingida a partir do percentual de respostas positivas, 
obtido pelo cálculo da combinação das duas categorias mais altas de resposta em cada dimensão. Valores percentuais mais altos indicam atitudes positivas em relação à cultura de segurança do paciente ${ }^{(17)}$.

Após a assinatura do Termo de Consentimento Livre e Esclarecido, os participantes foram instruídos quanto aos objetivos do estudo e os instrumentos preenchidos foram depositados em uma urna separadamente, sem identificação, exceto os dados demográficos do HSOPSC.

Os dados sociodemográficos foram analisados por meio de estatística descritiva, sendo realizada apuração de frequências, tanto em termos absolutos quanto em percentuais para cada categoria dos profissionais de enfermagem.

Para a análise e interpretação dos resultados foram utilizadas as orientações da $A H R Q^{(17)}$. Estas incluem a combinação das duas categorias mais altas de resposta (concordo fortemente/concordo e na maioria das vezes/sempre), para os itens redigidos positivamente e as duas categorias mais baixas de resposta, (discordo fortemente/ discordo e nunca/raramente) para itens redigidos negativamente ou reversos. Depois disso o percentual de cada item foi calculado. Valores percentuais mais altos indicam atitudes positivas em relação à cultura de segurança do paciente. Áreas de força para a segurança do paciente são definidas pela AHRQ para os itens ou dimensões em que mais de $75 \%$ dos entrevistados responderam com concordo fortemente/concordo e na maioria das vezes/sempre, para os itens redigidos positivamente, ou com discordo fortemente/ discordo e nunca/raramente, para itens redigidos negativamente. Áreas com potencial de melhoria são identificadas como os itens ou dimensões com percentuais de resposta positiva abaixo de $50 \%$, enquanto que as áreas neutras são aquelas em que o percentual de respostas positivas foi inferior a $75 \%$ e superior a $50 \%$.

Os percentuais de avaliação da cultura de segurança foram calculados para cada uma das UTI e de maneira global, sendo apresentados em gráfico. $O$ teste $Z$ para duas proporções foi usado para testar a existência de diferenças significativas entre as percentagens de respostas positivas das dimensões da cultura de segurança entre as duas UTI. Foi utilizado o Coeficiente de Correlação de Pearson ( $r$ ) para verificar a existência de correlação entre as dimensões sendo adotados os seguintes critérios: <0,3 (fraca correlação); >0,3 a <0,5 (moderada correlação) e >0,5 (forte correlação).

\section{RESULTADOS}

O Gráfico 1 apresenta a avaliação das dimensões da cultura de segurança do paciente da UTI1 e UTI2 e das duas UTI. Os escores representam o percentual de respostas positivas obtidas para cada dimensão. $O$ cálculo da média de escores positivos das 12 dimensões resultou 34,7\% no geral, 34,2\% para a UTI 1 e 35,2\% para UTI 2. No geral (UTI1 e UTI2), as dimensões com maiores percentuais foram: trabalho em equipe dentro das unidades (62,8\%), expectativas e ações de promoção da segurança do paciente do supervisor/gerente $(49,1 \%)$ e aprendizado organizacional, melhoria contínua $(46,1 \%)$. As dimensões com menores percentuais foram: apoio da gestão hospitalar para a segurança do paciente $(13,6 \%)$, respostas não punitivas aos erros $(17,4 \%)$ e percepção geral de segurança do paciente $(25,9 \%)$. 


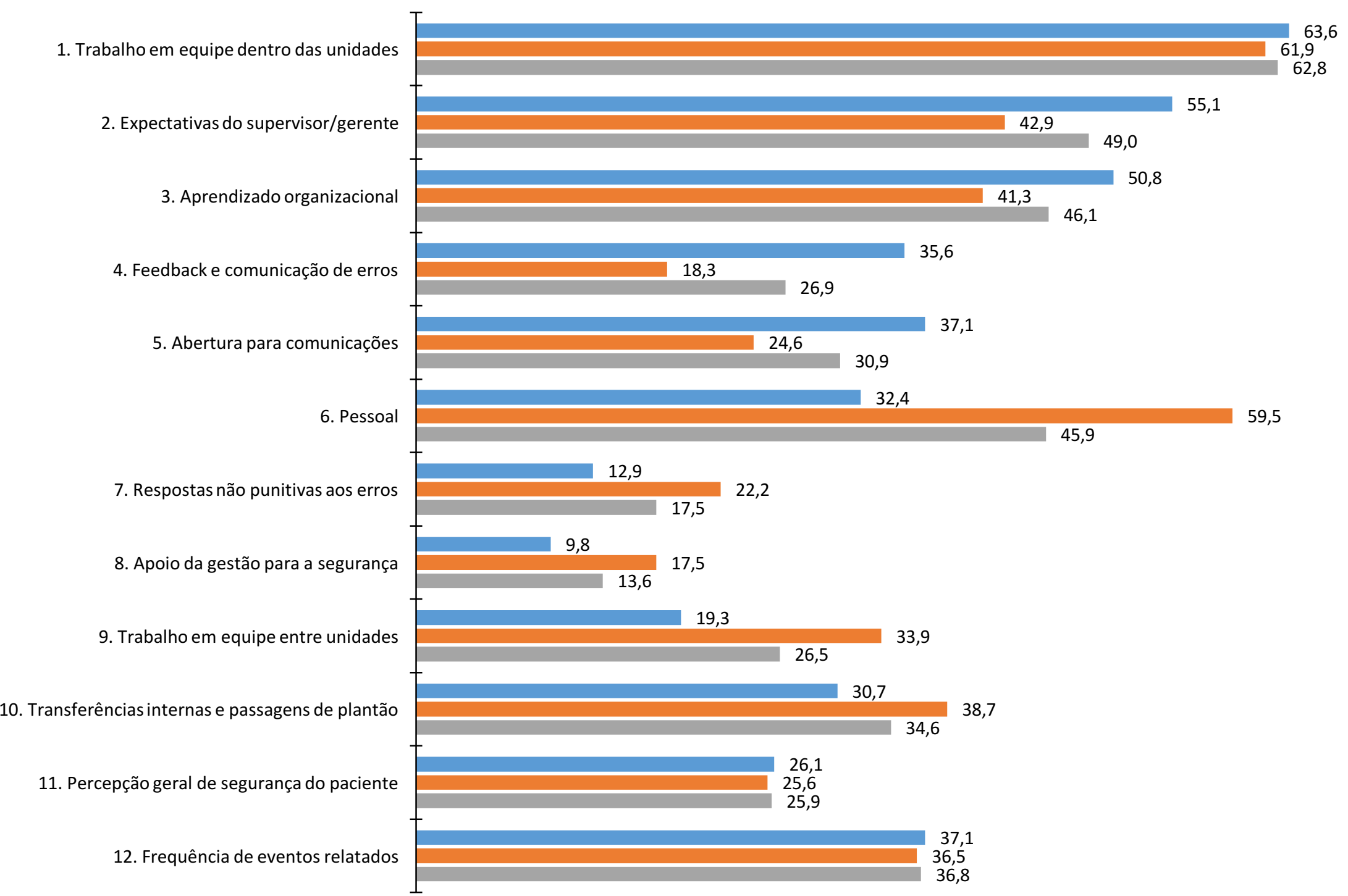


O Gráfico 1 também exibe os resultados do teste Z para avaliar diferenças entre os percentuais de resposta positiva das duas UTI. Este mostrou que não houve diferenças significativas entre as duas UTI para P-valor $=10 \%$, exceto para as dimensões 6 e 4 .

O grau de segurança do paciente, avaliado por categoria profissional de enfermagem e em geral, é apresentado no Gráfico 2. A maioria dos profissionais $(77,7 \%)$ classifica a segurança como muito boa/aceitável. Técnicos e auxiliares de enfermagem apresentaram melhores avaliações (boa/aceitável $81,7 \%$ e $85,6 \%$ ) do que os enfermeiros (aceitável/fraca - 78,9\%).

Enfermeiro $\quad$ Técnico $\square$ Auxiliar Geral

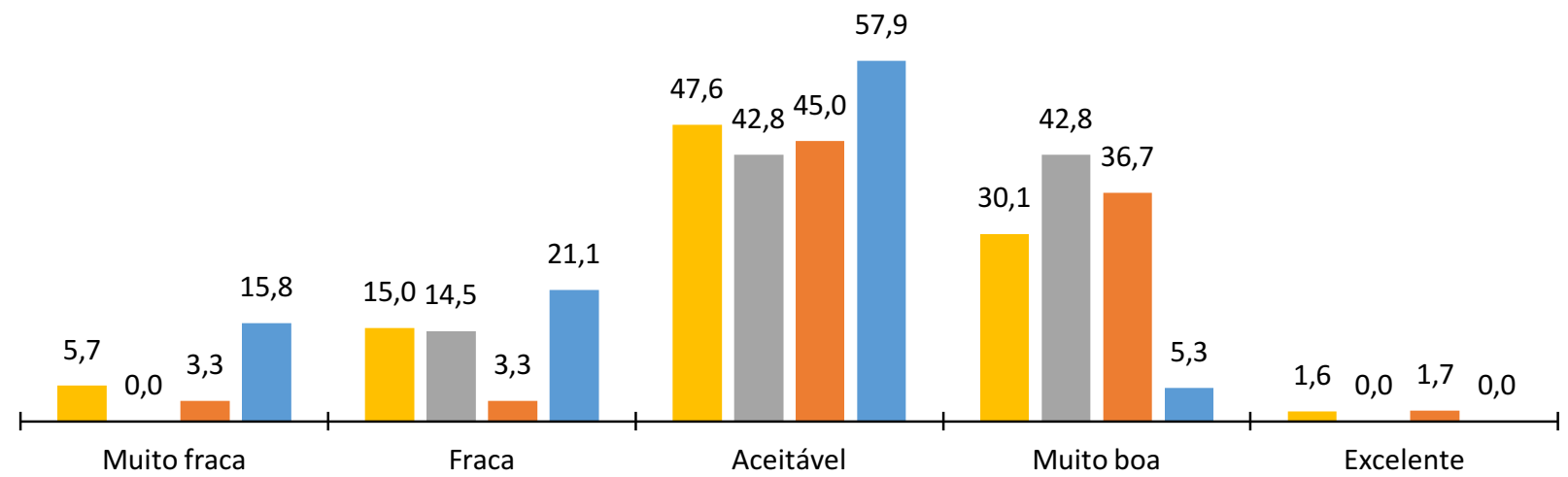

Gráfico 2: Avaliação do grau de segurança do paciente por categoria profissional de enfermagem e em geral de duas Unidades de Terapia Intensiva do sul do Brasil, 2011.

As respostas referentes ao número de eventos comunicados a chefia imediata nos últimos 12 meses conforme as diferentes categorias de enfermagem e globalmente, são apresentadas no Gráfico 3 . Seus resultados demonstram que poucos eventos são comunicados, pois a maioria das respostas foi nenhum $(30,2 \%)$ e de um a dois eventos $(37,2 \%)$. Quanto a categoria profissional, os enfermeiros informam mais os eventos, pois $79 \%$ informaram de um a cinco eventos, sendo que técnicos, a maioria (76,9\%) comunicou no máximo dois eventos. Porém 42,8\% dos auxiliares informaram de 11 a 20 eventos.

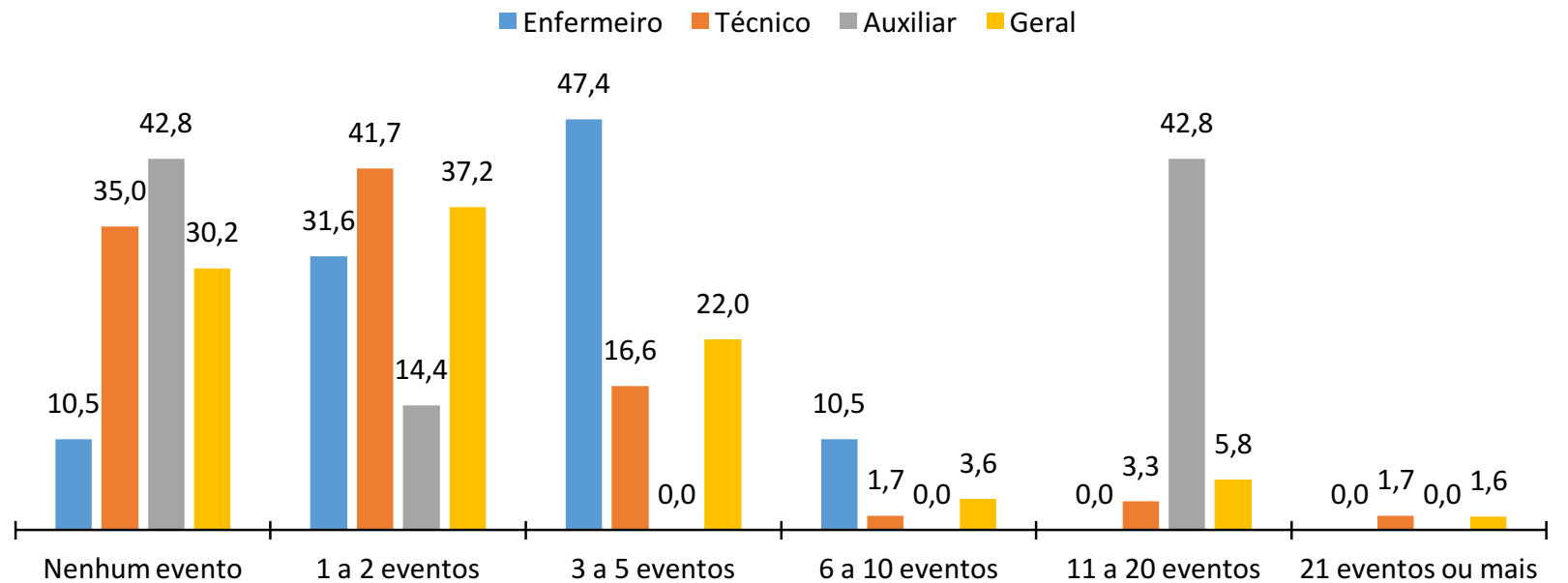

Gráfico 3: Número de eventos comunicados a chefia imediata nos últimos 12 meses por categoria profissional de enfermagem e em geral de duas Unidades de Terapia Intensiva do sul do Brasil, 2011. 
A Tabela 1 exibe os resultados das correlações entre as dimensões grau de segurança do paciente, número de eventos comunicados nos últimos 12 meses e os itens das dimensões percepção geral de segurança do paciente (A10R, A15, A17R, A18) e frequência de eventos relatados (D1, D2, D3).

Tabela 1: Correlações entre as dimensões grau de segurança do paciente, número de eventos comunicados, percepção geral de segurança do paciente e frequência de eventos relatados de duas Unidades de Terapia Intensiva do sul do Brasil, 2011.

\begin{tabular}{|c|c|c|c|c|c|c|c|c|c|}
\hline & $\begin{array}{c}\text { Grau de Segurança } \\
\text { paciente }\end{array}$ & $\begin{array}{c}\text { Número de } \\
\text { eventos }\end{array}$ & A10R & A15 & A17R & A18R & D1 & D2 & D3 \\
\hline $\begin{array}{c}\text { Grau segurança } \\
\text { do paciente }\end{array}$ & 1 & & & & & & & & \\
\hline $\begin{array}{c}\text { Números de } \\
\text { eventos }\end{array}$ & 0.023 & 1 & & & & & & & \\
\hline A10R & 0.290 & -0.083 & 1 & & & & & & \\
\hline A15 & 0.388 & -0.086 & 0.205 & 1 & & & & & \\
\hline A17R & -0.513 & 0.150 & -0.320 & -0.541 & 1 & & & & \\
\hline $\mathrm{A} 18 \mathrm{R}$ & 0.498 & 0.026 & 0.256 & 0.343 & -0.429 & 1 & & & \\
\hline D1 & 0.456 & 0.087 & 0.003 & 0.356 & -0.205 & 0.484 & 1 & & \\
\hline D2 & 0.389 & 0.106 & 0.091 & 0.261 & -0.199 & 0.488 & 0.838 & 1 & \\
\hline D3 & 0.314 & 0.122 & 0.179 & 0.271 & -0.204 & 0.391 & 0.651 & 0.742 & 1 \\
\hline
\end{tabular}

Legenda:

- A10R: Erros mais graves não acontecem aqui por acaso;

- A15: A segurança do paciente nunca é sacrificada para que mais trabalho seja feito;

- A17R: Nós temos problemas de segurança do paciente na unidade;

- A18R: Nossos processos e sistemas são adequados para prevenir a ocorrência de erros;

- D1: Quando um erro é cometido, mas o é percebido e corrigido antes de afetar o paciente, com que frequência é comunicado?;

- D2: Quando um erro é cometido, mas não tem potencial para prejudicar o paciente, com qual frequência é comunicado?;

- D3: Quando ocorre um erro que poderia prejudicar o paciente, mas o paciente não é afetado, com que frequência é comunicado?

Esses resultados mostram correlação moderada e negativa entre o item "nós temos problemas de segurança do paciente nesta unidade" (A17R) e o grau de segurança do paciente $(r=-0,513)$. Observa-se correlação positiva e moderada a baixa entre o item "nossos procedimentos e sistemas são adequados para prevenir a ocorrência de erros" (A18) e o grau de segurança do paciente $(r=0,498)$. O item (D1) também demonstrou correlação positiva e moderada a baixa com a avaliação do grau de segurança $(r=0,456)$. Já entre os itens da dimensão frequência de eventos relatados (D1 e D2, r=0,838; D1e D3, r=0,651; D2 e D3, r=0,742) foram encontradas correlações moderadas a fortes e positivas.

\section{DISCUSSÃO}

Os resultados deste estudo revelam uma cultura de segurança frágil na maioria das dimensões. Sua média percentual de $34,83 \%$ de respostas positivas para as 12 dimensões contrasta com outros estudos realizados em UTI, com percentuais de $62 \%^{(17)}, 55,24 \%^{(18)}$. Também apresenta avaliação inferior a estudo realizado em Unidades de Terapia Intensiva neonatais do Brasil com percentual de $42,58 \%{ }^{(19)}$.

Essa avaliação suscita a necessidade de mudanças nos vários aspectos da cultura de segurança do paciente nas instituições onde o estudo foi realizado, que podem ser priorizadas a partir das dimensões com avaliações menos positivas.

A dimensão apoio da gestão hospitalar para a segurança do paciente obteve menor percentual de 
avaliação positiva (13,6\%), evidenciando que a maioria dos profissionais percebe que a administração não propicia um clima de trabalho que favoreça a segurança. Outros estudos apresentaram resultados similares, com $22 \%$ no Brasil ${ }^{(19)}$ e $26,3 \%$ na Noruega ${ }^{(18)}$. Por outro lado, estudo da $\mathrm{AHRQ}^{(17)}$ apresentou um percentual de $63 \%$ de respostas positivas para este item.

Nas últimas décadas, gestores hospitalares enfrentam aumento da responsabilização pela qualidade e segurança dos cuidados. Diante disso, novas estruturas de administração, educação e avaliação da qualidade são necessárias, porém não são suficientes para alcançar a melhoria desejada. Assim, muitos gestores estão descobrindo que este desafio requer um novo nível de colaboração com a equipe de saúde da organização com esforços compartilhados para melhorar o atendimento ${ }^{(20)}$.

Líderes podem influenciar diretamente a qualidade e a segurança quando estabelecem metas e estratégias para melhoria do atendimento; apoiam e promovem uma cultura de melhoria da qualidade; desenvolvem lideranças, gerenciam resistências e planejam recursos para qualidade ${ }^{(21)}$.

Atualmente um dos maiores desafios em segurança do paciente é a assimilação pelos dirigentes de instituições de saúde da multifatorialidade dos erros e eventos adversos e que profissionais de saúde estão suscetíveis a cometer erros quando os processos são complexos e mal planejados ${ }^{(22)}$.

A resposta não punitiva ao erro, também com baixo percentual de avaliação positiva neste estudo $(17,5 \%)$, demonstra que ainda prevalece entre os profissionais o receio de que seus erros possam ser usados contra eles. Isso ressalta a importância da promoção de um ambiente aberto e não ameaçador para a comunicação de erros na assistência em saúde. Essa abordagem justa e equilibrada contribui para um nível maior de notificação de eventos, adesão a melhores práticas de segurança e consequentemente a redução de erros ${ }^{(23)}$.

A avaliação de $31 \%$ para a dimensão abertura para comunicação, sinaliza a premência de mudanças que promovam um ambiente no qual seja possível falar livremente sobre os aspectos que podem afetar negativamente o paciente e comunicar erros e eventos adversos sem o temor da punição.

Com esse estudo também foi observado, pela dimensão frequência de eventos relatados e pelo questionamento sobre o número de eventos comunicados a chefia, uma baixa taxa de relatos de erros entre os profissionais. Ficou evidente que quando há a tendência do profissional de informar o erro, este informa desde o erro mais simples e que é corrigido antes de acontecer, até o erro que efetivamente ocorreu, aspecto mostrado pela forte correlação entre as variáveis do estudo destinadas a essa avaliação.

A dimensão feedback e comunicação de erros, cuja avaliação foi de $27,1 \%$, destaca a necessidade de os profissionais saberem sobre os erros e/ou eventos adversos que foram informados e as medidas implementadas. Assim, a percepção de situações de risco pelo conhecimento do que acontece na instituição, poderá colaborar para o adequado gerenciamento do cuidado com enfoque na prevenção do erro e o estabelecimento da cultura de segurança ${ }^{(24)}$.

Durante a realização deste estudo as iniciativas em segurança do paciente eram incipientes no Brasil e a notificação de erros e eventos adversos não era uma prática formalizada nas instituições do estudo, 
podendo justificar os baixos percentuais de respostas positivas destas dimensões.

Esta situação também pode influenciar a percepção dos profissionais acerca da segurança do paciente. O presente estudo, embora aponte uma boa avaliação nas respostas ao grau de segurança $(77,7 \%$ - muito boa/aceitável), revelou uma baixa avaliação positiva para a dimensão percepção geral de segurança do paciente $(25,9 \%)$. Estudo em UTI neonatais no Brasil ${ }^{(19)}$, também apresentou resultados similares, com avaliação do grau de segurança como muito boa e aceitável por $83 \%$ dos profissionais e da dimensão percepção geral de segurança do paciente com percentual de $36 \%$ de respostas positivas.

A correlação moderada e negativa encontrada entre os itens "nós temos problemas de segurança do paciente nesta unidade" (A17R) e o grau de segurança do paciente $(r=0,513)$ significa que os profissionais que mais concordam que há problemas de segurança do paciente, apresentaram pior avaliação do grau de segurança. Por outro lado, se os profissionais têm percepções positivas sobre os procedimentos e sistemas para a prevenção de erros, sua avaliação do grau de segurança do paciente será positiva, e vice-versa.

Ainda sobre a avaliação do grau se segurança, foi evidenciado que quando o profissional tende a comunicar mais os erros que são cometidos, mas percebidos e corrigidos antes de afetar o paciente, há uma percepção melhor do grau de segurança por esse profissional, ou ao contrário, se ele comunica menos, suas avaliações do grau de segurança são piores.

Embora a cultura de segurança possa variar entre instituições diferentes ou até mesmo entre unidades de uma mesma instituição, a peculiaridade do trabalho e características do ambiente de terapia intensiva podem indicar similaridades.

De fato, foram observadas semelhanças entre as avaliações dos profissionais das duas UTI, especialmente para trabalho em equipe, percepção geral de segurança, frequência de eventos relatados dentre outras. Entretanto, diferenças entre as UTI foram estatisticamente comprovadas para duas dimensões, ou seja, pessoal e feedback e comunicação de erros. Essa informação sugere a necessidade da UTI1 em relação a UTI2, de priorizar melhorias em relação a quantitativo de pessoal pois os itens dessa dimensão com escore mais baixo foram "o quadro de pessoal é suficiente para a carga de trabalho da unidade" (20\%) e "trabalhamos em modo de crise, tentando fazer muita coisa, muito depressa" (16\%). Já na UTI2 a prioridade consiste na implementação de mudanças nos processos de comunicação, especialmente a respeito de erros, tendo em vista os baixos percentuais de respostas positivas aos itens "nós somos informados sobre os erros que acontecem nesta unidade" (14\%) e "nesta unidade, discutimos maneiras para prevenir que os erros aconteçam novamente" (19\%).

Muito se tem discutido que o quantitativo de pessoal representa um importante indicador de segurança do paciente. Pesquisa conduzida em UTI de dois hospitais brasileiros ${ }^{(25)}$ demonstrou que pacientes assistidos por profissionais de enfermagem sobrecarregados tiveram mais do que o dobro de chance de sofrerem ao menos um evento adverso. Apontou também que as complicações atribuídas à sobrecarga de trabalho de enfermagem aumentaram o número de dias de internação e o risco de mortalidade dos pacientes. 
A maioria das dimensões avaliadas nas UTI1 e 2 não apresentaram resultados consistentes com uma cultura de segurança, conforme limite de $75 \%$ estabelecido pela $A H R Q^{(17)}$. Apesar disso, foram identificados alguns itens com percepções favoráveis dentre os demais, tais como trabalho em equipe e expectativas do supervisor/gerente.

Na dimensão trabalho em equipe, o item que mais se destacou, com $92 \%$ de respostas positivas, foi “quando há muito trabalho que precisa ser realizado rapidamente, trabalhamos juntos, em equipe, para que o trabalho seja feito" e na dimensão expectativas do supervisor/gerente ficaram em destaque os itens reversos "sempre que a pressão no trabalho aumenta, a minha chefia quer que trabalhemos mais rápido, mesmo que isso signifique usar atalhos" (65\%) e "minha chefia considera seriamente as sugestões dos profissionais para melhorar a segurança do paciente" (58\%).

Reforçando a importância desse resultado, a literatura destaca que o trabalho em equipe eficaz é crucial para fornecer um cuidado ideal ao paciente e que a liderança é vital para orientar a maneira pela qual os membros da equipe da UTI devem interagir, coordenar-se uns com os outros ${ }^{(4)}$ bem como desenvolver as atividades de forma segura.

\section{CONCLUSÃO}

Este estudo possibilitou uma avaliação geral da segurança percebida pelos profissionais de enfermagem de duas UTI. Os resultados indicam que a cultura de segurança ainda precisa ser melhor desenvolvida nos locais do estudo, pois todas as dimensões necessitam de melhorias. No entanto, mais atenção deve ser dedicada ao apoio da gestão para a segurança, erros e percepção geral de segurança e respostas não punitivas aos erros, e sob este aspecto, os resultados do estudo apontam uma lacuna para a necessidade de estratégias futuras de desenvolvimento de educação permanente que melhore o resultado encontrado nestas dimensões, e que também desencadeie novos estudos.

Ao realizar uma análise de correlação entre as diferentes dimensões, o estudo mostrou uma interpretação inovadora, geralmente não observada na análise tradicional do instrumento que considera pontos fortes e fracos das instituições.

Os resultados constituem um diagnóstico útil para que gestores dessas instituições desenvolvam estratégias eficazes na melhoria da qualidade dos cuidados de saúde para garantir a segurança do paciente. Podem ser utilizados como referência em estudos futuros, avaliando o resultado de intervenções implementadas com base nos presentes resultados. Representa uma singularidade cultural que deve ser levada em consideração sempre que ferramentas de medição da cultura de segurança são aplicadas em diferentes configurações de cultura.

Certas limitações precisam ser reconhecidas no presente estudo. Primeiro, o instrumento de avaliação da cultura de segurança utilizado não tinha sido ainda validado no Brasil, sendo apenas traduzido e aplicado nos locais do estudo o que poderia comprometer a sua confiabilidade e consistência interna. Outra limitação foi a realização do estudo somente com profissionais de enfermagem. Portanto, estes resultados podem não 
refletir a cultura de segurança do paciente sob a ótica de outros profissionais de saúde. Assim, mais pesquisas são necessárias, utilizando o instrumento já validado com os diversos profissionais de saúde, unidades e instituições hospitalares.

\section{REFERÊNCIAS}

1. Kohn KT, Corrigan JM, Donaldson MS. To err is human: building a safer health system [Internet]. Washington: National Academies Press (US); 2000 [acesso em: 04 mai. 2017]. Disponível em: http://dx.doi.org/10.17226/9728.

2. Lamont T, Waring J. Safety lessons: shifting paradigms and new directions for patient safety research. J Health Serv Res Policy [Internet]. 2015 [acesso em: 04 mai. 2017];20(1 Supl.):1-8. Disponível em:

http://dx.doi.org/10.1177/1355819614558340.

3. Wachter RM, Pronovost P, Shekelle P. Strategies to improve patient safety: the evidence base matures. Ann Intern Med [Internet]. 2013 [acesso em: 04 mai. 2017];158(5 Part 1):350-2. Disponível em: http://dx.doi.org/10.7326/00034819-158-5-201303050-00010.

4. Oliveira RM, Leitão IMTA, Silva LMS, Figueiredo SV, Sampaio RL, Gondim MM. Strategies for promoting patient safety: from the identification of the risks to the evidence-based practices. Esc Anna Nery [Internet]. 2014 [acesso em: 04 mai. 2017];18(1):122-9. Disponível em: http://dx.doi.org/10.5935/1414-8145.20140018.

5. Leape LL. Patient Safety in the Era of Healthcare Reform. Clin Orthop Relat Res [Internet]. 2015 [acesso em: 04 mai. 2017];473(5):1568-73. Disponível em: http://dx.doi.org/10.1007/s11999-014-3598-6.

6. Singer S, Lin S, Falwell A, Gaba D, Baker L. Relationship of safety climate and safety performance in hospitals. Health Serv Res [Internet]. 2009 [acesso em: 04 mai. 2017];44(2 Pt 1):399-421. Disponível em: http://dx.doi.org/10.1111/j.1475-6773.2008.00918.x.

7. DiCuccio MH. The Relationship Between Patient Safety Culture and Patient Outcomes: A Systematic Review. J Patient Saf [Internet]. 2015 [acesso em: 04 mai. 2017];11(3):135-42. Disponível em:

http://dx.doi.org/10.1097/PTS.0000000000000058.

8. Najjar S, Nafouri N, Vanhaecht K, Euwema M. The relationship between patient safety culture and adverse events: a study in palestinian hospitals. Saf Heal [Internet]. 2015 [acesso em: 04 mai. 2017];1(1):16. Disponível em:

http://dx.doi.org/10.1186/s40886-015-0008-z.

9. Kaufman G, McCaughan D. The effect of organisational culture on patient safety. Nurs Stand [Internet]. 2013 [acesso em: 04 mai. 2017];27(43):50-6. Disponível em: http://dx.doi.org/10.7748/ns2013.06.27.43.50.e7280.

10. Stavrianopoulos T. The development of patient safety culture. Health Science Journal [Internet]. 2012 [acesso em: 04 mai. 2017];6(2):201-11. Disponível em: http://www.hsj.gr/medicine/the-development-of-patient-safetyculture.php?aid=3262.

11. Ulrich B, Kear T. Patient Safety and Patient Safety Culture: Foundations of Excellent Health Care Delivery. Nephrol Nurs J. 2014;41(5):447-56.

12. Halligan M, Zecevic A. Safety culture in healthcare: a review of concepts, dimensions, measures and progress. BMJ Qual Saf [Internet]. 2011 [acesso em: 04 mai. 2017];20(4):338-43. Disponível em:

http://dx.doi.org/10.1136/bmjqs.2010.040964.

13. Nieva VF, Sorra J. Safety culture assessment: a tool for improving patient safety in healthcare organizations. Qual Saf Heal Care [Internet]. 2003 [acesso em: 04 mai. 2017];12(suppl 2):ii17 LP-ii23. Disponível em:

http://dx.doi.org/10.1136/qhc.12.suppl_2.ii17.

14. Reis CT, Laguardia J, Martins M. Adaptação transcultural da versão brasileira do Hospital Survey on Patient Safety Culture: etapa inicial. Cad Saude Publica [Internet]. 2012 [acesso em: 04 mai. 2017];28(11):2199-210. Disponível em: http://dx.doi.org/10.1590/S0102-311X2012001100019.

15. Mello JF, Barbosa SFF. Cultura de segurança do paciente em terapia intensiva: recomendações da enfermagem.

Texto contexto - enferm [Internet]. 2013 [acesso em: 04 mai. 2017];22(4):1124-33. Disponível em: http://dx.doi.org/10.1590/S0104-07072013000400031.

16. Jackson J, Sarac C, Flin R. Hospital safety climate surveys: measurement issues. Curr Opin Crit Care [Internet]. 2010 [acesso em: 04 mai. 2017];16(6):632-8. Disponível em: http://dx.doi.org/10.1097/MCC.0b013e32833f0ee6. 
17. Agency for Healthcare Research and Quality. 2014 User Comparative Database Report | Agency for Healthcare Research \& Quality [Internet]. Rockville, MD: . Agency for Healthcare Research and Quality; 2014 [atualizado em: 28 mar. 2014; acesso em: 04 mai. 2017]. Disponível em: http://www.ahrq.gov/professionals/quality-patientsafety/patientsafetyculture/hospital/2014/index.html.

18. Ballangrud R, Hedelin B, Hall-Lord ML. Nurses' perceptions of patient safety climate in intensive care units: a crosssectional study. Intensive Crit Care Nurs [Internet]. 2012 [acesso em: 04 mai. 2017];28(6):344-54. Disponível em: http://10.1016/j.iccn.2012.01.001.

19. Tomazoni A, Rocha PK, Souza S, Anders JC, Malfussi HFC. Patient safety culture at neonatal intensive care units: perspectives of the nursing and medical team. Rev Lat Am Enfermagem [Internet]. 2014 [acesso em: 04 mai. 2017];22(5):755-63. Disponível em: http://dx.doi.org/10.1590/0104-1169.3624.2477.

20. Goeschel CA, Wachter RM, Pronovost PJ. Responsibility for quality improvement and patient safety: hospital board and medical staff leadership challenges. Chest [Internet]. 2010 [acesso em: 04 mai. 2017];138(1):171-8. Disponível em: http://dx.doi.org/10.1378/chest.09-2051.

21. Parand A, Dopson S, Renz A, Vincent C. The role of hospital managers in quality and patient safety: a systematic review. BMJ Open [Internet]. 2014 [acesso em: 04 mai. 2017];4(9):e005055. Disponível em:

http://dx.doi.org/10.1136/bmjopen-2014-005055.

22. Silva AEBC. Segurança do paciente: desafios para a prática e a investigação em Enfermagem. Rev. Eletr. Enf. [Internet]. 2010 [acesso em: 04 mai. 2017];12(3):422. Disponível em: http://dx.doi.org/10.5216/ree.v12i3.11885. 23. Petschonek S, Burlison J, Cross C, Martin K, Laver J, Landis RS, Hoffman JM. Development of the just culture assessment tool: measuring the perceptions of health-care professionals in hospitals. J Patient Saf [Internet]. 2013 [acesso em: 04 mai. 2017];9(4):190-7. Disponível em: https://dx.doi.org/10.1097\%2FPTS.0b013e31828fff34. 24. Duarte SCM, Stipp MAC, Silva MM, Oliveira FT. Eventos adversos e segurança na assistência de enfermagem. Rev Bras Enferm [Internet]. 2015 [acesso em: 04 mai. 2017];68(1):144-54. Disponível em: http://dx.doi.org/10.1590/00347167.2015680120p.

25. Novaretti MCZ, Santos EV, Quitério LM, Daud-Gallotti RM. Sobrecarga de trabalho da Enfermagem e incidentes e eventos adversos em pacientes internados em UTI. Rev Bras Enferm [Internet]. Rev Bras Enferm [Internet]. 2014 [acesso em: 04 mai. 2017];67(5):692-9. Disponível em: http://dx.doi.org/10.1590/0034-7167.2014670504. 